\title{
Intermittency effects in single- and multi-phase flow and anomalous transport in heterogeneous porous media
}

\author{
Zoë Penko ${ }^{1}$, Yaofa Li ${ }^{1,2}$, Diogo Bolster ${ }^{1,3}$, and Kenneth T. Christensen ${ }^{1,3,4}$ \\ ${ }^{1}$ University of Notre Dame, Department of Aerospace and Mechanical Engineering \\ ${ }^{2}$ Now at Montana State University, Department of Mechanical and Industrial Engineering \\ ${ }^{3}$ University of Notre Dame, Department of Civil and Environmental Engineering and Earth Sciences \\ ${ }^{4}$ Now at Illinois Institute of Technology, Armour College of Engineering
}

\section{Introduction}

Multi-phase flow and transport in porous media is prevalent in a wide range of challenging fluid mechanics problems related to sustainability, energy, and the environment. Accurate prediction of the displacement and interaction of such flows is vital in addressing these problems. In particular it is critical to understand the small- or pore-scale flow and its spatial and temporal evolution, which can impact behaviors at system scales in a nontrivial manner. Intermittency is a phenomenon currently observed in numerical and experimental studies of single-phase flow (Anna et al., n.d.; Morales et al., n.d.), but the case of multi-phase flow has yet to receive much study due to challenges faced in both simulations and experiments. The underlying physics of spreading, mixing, and interfacial processes must be understood for accurate predictions of transport in multi-phase flow systems. Therefore, a comprehensive understanding of multi-phase flow at these very small scales is necessary in the development of accurate system-scale prediction models. We present results from a coordinated numerical and experimental study of intermittency effects over a range of viscous and inertial flow regimes in single- and multi-phase flows in $2 \mathrm{D}$ heterogeneous micromodels to quantify Lagrangian flow statistics to better inform pore-scale models. The applicability of different modeling frameworks such as the correlated-continuous time random walk is tested by studying statistics of particle trajectories obtained by particle tracking velocimetry (PTV) measurements and Lattice Boltzmann simulations from single- and multi-phase flows. The results make particular note of the influence of the pore Reynolds number $(R e)$ and inertial effects on intermittency, and compare these effects in the two flow regimes.

\section{Methods}

Experiments are conducted in the University of Notre Dame MicroPIV Laboratory for Energy and the Environment. A PTV approach utilizing small, neutrally-buoyant (to serve as true tracers), and low-density seeded particles illuminated by laser light pulses synced to a camera by a synchronizer that captures images of the fluoresced light to reconstruct particle trajectories of single particles is used. Volume illumination is employed to excite the fluorescent tracer particles seeded in the flow and the particle trajectories are imaged in a plane-defined $2 \mathrm{D}$ micromodel depth. Figure 1 presents homogeneous and heterogeneous $2 \mathrm{D}$ porous models that are fabricated using established silicon microfabrication methods (Kazemifar, 2020), where the top of the micromodel is formed by anodic bonding of a glass wafer to provide optical access to the flow in the $2 \mathrm{D}$ porous matrix.

Some limitations exist in collecting quality experimental data, as higher Re flow experiments pose challenges due to the inherent trade-off between spatial resolution and the physical size of the field of view (FOV), image acquisition rate limitation 


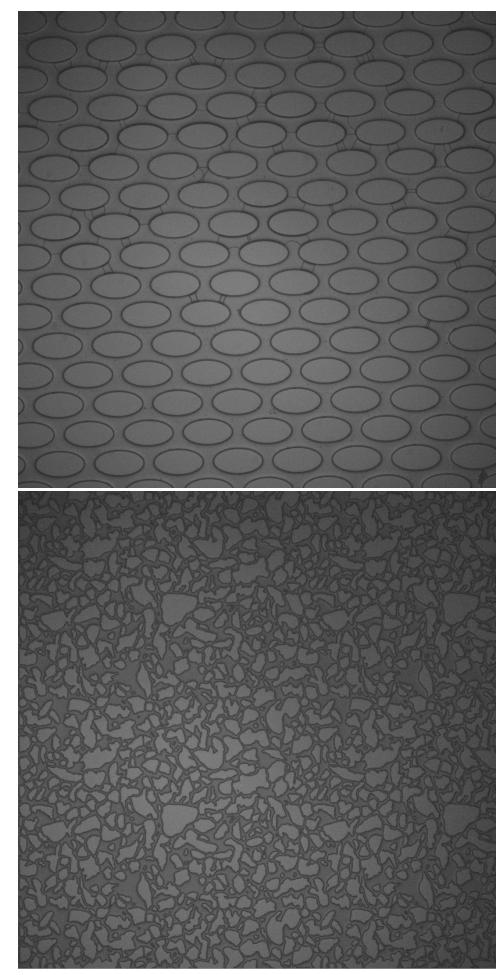

Figure 1: (top) Homogeneous 2D porous micromodel; (bottom) Heterogeneous 2D porous micromodel.

by data transfer bandwidth, and the record length limitation by the on-board memory size for a given resolution. Such limitations can be addressed by a Lattice Boltzmann method (LBM) simulation approach to numerically simulate multi-phase flow in the same porous media and complement experiments by broader parameter space exploration, an approach used on a 2D micromodel of water (wetting phase) being displaced by $\mathrm{CO}_{2}$ (non-wetting phase) by Li et al. (n.d.).

\section{Future Work}

A coordinated simulation and experimental study of intermittency effects in multi-phase flow spanning flow regimes from viscous to inertial in $2 \mathrm{D}$ and $3 \mathrm{D}$ porous media is underway in an effort to quantify Lagrangian flow statistics and accurately model the pore-scale behavior, thereby advancing the fundamental understanding of such behavior. These data will be used to (1) calculate statistics of particle trajectories and intermittency for singlephase flow in 2D porous micromodels to test the applicability of different modeling frameworks, (2) study intermittency and dispersion with the addition of a second, immiscible fluid and the presence of trapped ganglia, (3) study the effect of $R e$ and inertia on intermittency in multi-phase porous media flow, and (4) compare these effects in single- and multi-phase flow in homogeneous and heterogeneous porous models. This analysis will form the basis of the final paper. The development of a more general and robust modeling approach that is fully validated by experiments and simulations will allow better prediction of the spreading, mixing, and interfacial processes that occur in natural and engineered environments.

\section{References}

Anna, P. de, Le Borgne, T., Dentz, M., et al. (n.d.). Flow Intermittency, Dispersion, and Correlated Continuous Time Random Walks in Porous Media. Physical Review Letters 110, (), 184502.

Kazemifar, F. (2020). An experimental study of supercritical $\mathrm{CO}_{2}$ flow in pipes and porous micromodels for carbon sequestration applications (). URL: https : / / core . ac . uk / reader / 29175208 (visited on 07/16/2020).

Li, Y., Kazemifar, F., Blois, G., and Christensen, K. T. (n.d.). Micro-PIV measurements of multiphase flow of water and liquid $\mathrm{CO}_{2}$ in 2-D heterogeneous porous micromodels. Water Resources Research 53, (), 6178-6196. ISSN: 00431397.

Morales, V. L., Dentz, M., Willmann, M., and Holzner, M. (n.d.). Stochastic dynamics of intermittent pore-scale particle motion in threedimensional porous media: Experiments and theory. Geophysical Research Letters 44, (), 93619371. ISSN: 0094-8276. 